\title{
CMR imaging in patients with cardiac amyloidosis
}

C ardiac ATTR amyloidosis is an often fatal condition in which wild-type or mutated forms of transthyretin are deposited as amyloid in the heart. Although specific therapies for patients with this condition are emerging, diagnosis as well as monitoring of the progression of the disorder can be challenging. According to Marianna Fontana and colleagues from the UK, "a suggestive constellation of electrocardiography, echocardiography, and biomarker findings are found mainly in advanced disease, but interpretation may be confounded by common comorbidities, such as left ventricular hypertrophy". In 2013, the UK-based group reported that native myocardial $\mathrm{T} 1$ mapping on cardiac magnetic resonance (CMR) images was useful in the tracking of disease progression in patients with cardiac AL amyloidosis. Fontana et al. have now assessed whether this imaging technique could also prove useful in patients with cardiac ATTR amyloidosis.

Compared with healthy individuals $(n=52)$, patients with hypertrophic cardiomyopathy $(n=46)$, and patients with a pathogenic mutation in the gene for transthyretin, but no evidence of cardiac amyloid deposition $(n=8)$, T1 was elevated in 85 patients with cardiac ATTR amyloidosis. Notably, however, although native T1 mapping was similarly accurate for the diagnosis of ATTR and AL forms of cardiac amyloidosis, the T1 elevation in patients with cardiac ATTR amyloidosis was not as high as in individuals with AL amyloidosis $(n=79)$. The investigators describe this finding as "surprising", because ventricular wall thickness is typically greater in patients with the ATTR form than the AL form amyloidosis. T1 was found to increase with increasing burden of cardiac amyloid, and correlated with various measures of cardiac dysfunction in patients with cardiac ATTR or AL amyloidosis. The investigators concluded that "native myocardial T1 mapping detects cardiac ATTR amyloid and has similar performance for diagnosis

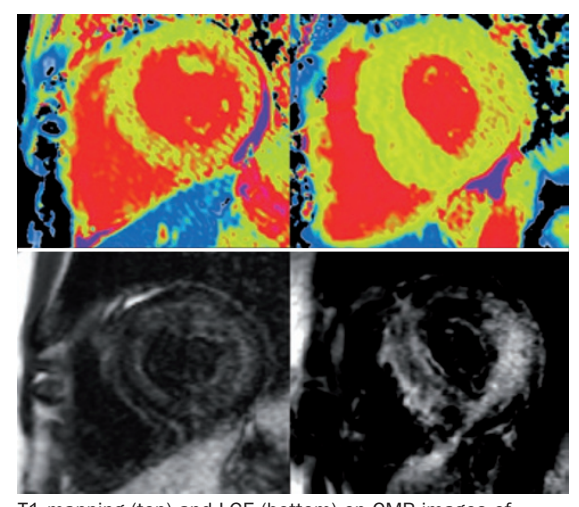

T1 mapping (top) and LGE (bottom) on CMR images of cardiac AL amyloidosis (left) and cardiac ATTR amyloidosis (right). Reprinted from Fontana, M. et al. Native T1 mapping in transthyretin amyloidosis. JACC Cardiovasc. Imaging doi:10.1016/j.jcmg.2013.10.008 (2014), with permission from the American College of Cardiology Foundation.

and tracking disease in both ATTR and AL amyloidosis".

In a separate study conducted in North America, a modified protocol for T1 mapping was performed on CMR images for 90 patients with suspected, but unconfirmed, cardiac amyloidosis (of various aetiology) and 64 patients with hypertensive left ventricular hypertrophy. Diffuse hyperenhancement was demonstrated in $53 \%$ of patients with suspected cardiac amyloidosis and in no patients with hypertensive left ventricular hypertrophy. Some hyperenhancement was found in an additional $12 \%$ of patients with suspected cardiac amyloidosis and in $6 \%$ of patients with hypertensive left ventricular hypertrophy. Diffuse or any hyperenhancement was strongly associated with all-cause mortality over a median follow-up time of 29 months.

Another group from the UK has also studied the use of CMR imaging in patients with cardiac amyloidosis and, in particular, whether ATTR amyloidosis can be differentiated from AL amyloidosis using this imaging modality. In this study, Jason Dungu and colleagues retrospectively compared late gadolinium enhancement (LGE) in 51 patients with cardiac ATTR amyloidosis and in 46 patients with cardiac AL amyloidosis. LGE was found in almost all patients with cardiac ATTR or AL amyloidosis (only one patient with AL amyloidosis did not have LGE). However, the pattern of LGE was more extensive in patients with cardiac ATTR amyloidosis, with all patients exhibiting right ventricular LGE (compared with $72 \%$ of those with AL amyloidosis) and the vast majority (90\%) exhibiting transmural LGE (compared with 37\%).

The investigators devised a score, known as the Query Amyloid Late Enhancement (QALE) score, for patients with suspected cardiac amyloidosis. The score includes LGE information for the base, midventricle, and apex of the heart, and was shown to be an independent predictor of amyloid type (OR 1.90, 95\% CI 1.2-3.0, $P=0.007)$. A logistic regression model that incorporated information on age, interventricular septum thickness, and the QALE score was found to have an $87 \%$ sensitivity and $96 \%$ specificity for the detection of cardiac ATTR amyloidosis.

In their study report, Dungu et al. acknowledge that "T1 mapping may become the gold standard for quantifying the extent of interstitial expansion by amyloid deposition", but point out that "it may not be possible to implement the technique into standard clinical practice for all patients, due to limited availability (CMR scanning is often performed locally before specialist center referral)". They conclude, therefore, that "CMR LGE scoring has the potential to enhance the management of many patients, but [given the retrospective nature of their study, and the lack of evaluation in a prospective cohort] cannot replace biopsy as the [current] gold standard."

\section{Bryony M. Mearns}

Original articles Fontana, M. et al. Native T1 mapping in transthyretin amyloidosis. JACC Cardiovasc. Imaging doi:10.1016/j.jcmg.2013.10.008 | White, J. A. et al. CMR imaging with rapid visual $\mathrm{T} 1$ assessment predicts mortality in patients suspected of cardiac amyloidosis. JACC Cardiovasc. Imaging doi:10.1016/j.jcmg.2013.09.019 | Dungu, J. N. et al. CMR-based differentiation of AL and ATTR amyloidosis. JACC Cardiovasc. Imaging doi:10.1016/ j.jcmg.2013.08.015 